\title{
A Search for Intra-group Planetary Nebulae in the M81 Group
}

\author{
John J. Feldmeier \\ Case Western Reserve University, 10900 Euclid Ave. Cleveland, OH \\ 44106 , U.S.A.
}

Patrick R. Durrell, Robin Ciardullo

Penn State University, 525 Davey Lab, University Park, PA 16802, U.S.A.

George H. Jacoby

WIYN Observatory, P.O. Box 26732, Tucson AZ 85726, U.S.A.

\begin{abstract}
We present the initial results from an [O III $] \lambda 5007$ survey for intra-group planetary nebulae in the M 81 group of galaxies. A total of 0.36 square degrees of the survey have been analyzed thus far, and a total of four intra-group candidates have been detected. These data allow us to probe the physics of galaxy interactions in small groups, and give us an upper limit for the density of intracluster starlight. We find that the M 81 group has less than $3 \%$ of its stars in an intra-group component; this is much less than the fraction seen in richer galaxy clusters.
\end{abstract}

Intracluster planetary nebulae (IPN) trace intracluster starlight, and provide crucial information on the kinematics of intracluster stars. However, although the presence of IPN in massive clusters such as Virgo and Fornax has now been well established (Feldmeier, these proceedings), the amount of intracluster planetaries in much poorer environments is unknown. Searching poorer systems for IPN is a promising, but currently unexplored, avenue of research. If most intracluster stars are removed by galaxy collisions (i.e., Richstone \& Malumuth 1983; Moore et al. 1996), the fraction of intra-group stars will be significantly less than that seen in a rich cluster.

As a first step, we have begun a large-scale [O III] $\lambda 5007$ IPN survey of the nearby M 81 group of galaxies. This galaxy group is known to be strongly interacting, with multiple tidal tails seen in H I gas (Yun, Ho, \& Lo 1994). Using the KPNO 4-meter telescope and the MOSAIC imager, we have observed 0.72 square degrees of the group, and we have analyzed the first 0.36 square degrees. Using our automated detection methods (i.e., Theuns \& Warren 1997; Feldmeier 2000), we found a total of 22 PN candidates in our first field, which can be divided into three components: 
- 16 PN candidates surrounding the elliptical galaxy NGC 3077, which is in the southeastern corner of the field.

- 2 PN that can be attributed to M 81's (NGC 3031) outer halo, which is directly west of this field.

- 4 intra-group PN candidates.

Of these four intra-group candidates, only a single object is above the photometric completeness level. If we assume this candidate is bona-fide, we can set an upper limit for the intra-group luminosity. In order to be conservative, we adopt a generous conversion factor between PN density and luminosity $\left(\alpha_{2.5}=16 \times 10^{-9} \mathrm{~L}_{\odot}\right)$, the amount derived for the bulge of M 81 (Jacoby et al. 1989). We find that, at most, there is $\sim 2 \times 10^{8} \mathrm{~L}_{\odot}$ of intra-group stars in our Mosaic field. When we compare this number to the total luminosity and angular area of the M 81 group (Garcia 1993), we find that less than 3\% of the group's stellar luminosity is intra-group in nature. This is much smaller than the $\sim 20 \%$ found for the Virgo and Fornax clusters (Feldmeier 2000; Durrell et al. 2001; Feldmeier et al. 2002). This result is consistent with tidal stripping scenarios.

Our PN survey is ongoing, and we will be able to place much more stringent limits on the intra-group luminosity surface density. We have also begun a broad-band survey for intra-group red giant stars in the M 81 group using the CFHT. The two methods complement each other: red giant surveys allow an independent check of our PN survey, and can probe to fainter surface brightnesses. On the other hand, through follow-up spectroscopy, IPN can provide dynamical information and nebular abundances. Finally, we also plan an ultra-deep imaging survey of the M81 group using the Burrell Schmidt. We will perform high-quality surface photometry to obtain the global picture of this system.

\section{References}

Durrell, P. R., Ciardullo, R., Feldmeier, J. J., Jacoby, G. H., \& Sigurdsson, S. 2001, ApJ, 570, 119

Feldmeier, J. J. 2000, Ph.D. Thesis, Penn State University

Feldmeier, J. J., Ciardullo, R., Krelove, K., Jacoby, G., \& Durrell, P. R. 2002, ApJ, in preparation

Garcia, A. M. 1993, A\&AS, 100, 47

Jacoby, G. H., Ciardullo, R., Ford, H. C., \& Booth, J. 1989, ApJ, 344, 70

Moore, B., Katz, N., Lake, G., Dressler, A., \& Oemler, A. 1996, Nature, 379, 613

Richstone D. O., \& Malumuth, E.M. 1983, ApJ, 268, 30

Theuns, T., \& Warren, S. J. 1997, MNRAS, 284, L11

Yun, M. S., Ho, P. T. P., \& Lo, K. Y. 1994, Nature, 372, 530 